\title{
Nonlinear Analysis for Shear Augmented Dispersion of Solutes in Blood Flow through Narrow Arteries
}

\author{
D. S. Sankar, ${ }^{1}$ Nurul Aini Binti Jaafar, ${ }^{2}$ and Yazariah Yatim $^{2}$ \\ ${ }^{1}$ School of Advanced Sciences, VIT University, Chennai Campus, Chennai 48, India \\ ${ }^{2}$ School of Mathematical Sciences, Universiti Sains Malaysia, 11800 Penang, Malaysia \\ Correspondence should be addressed to D. S. Sankar, sankar_ds@yahoo.co.in
}

Received 23 May 2012; Accepted 2 July 2012

Academic Editor: Turgut Öziş

Copyright (C) 2012 D. S. Sankar et al. This is an open access article distributed under the Creative Commons Attribution License, which permits unrestricted use, distribution, and reproduction in any medium, provided the original work is properly cited.

\begin{abstract}
The shear augmented dispersion of solutes in blood flow (i) through circular tube and (ii) between parallel flat plates is analyzed mathematically, treating blood as Herschel-Bulkley fluid model. The resulting system of nonlinear differential equations are solved with the appropriate boundary conditions, and the expressions for normalized velocity, concentration of the fluid in the core region and outer region, flow rate, and effective axial diffusivity are obtained. It is found that the normalized velocity of blood, relative diffusivity, and axial diffusivity of solutes are higher when blood is modeled by Herschel-Bulkley fluid rather than by Casson fluid model. It is also noted that the normalized velocity, relative diffusivity, and axial diffusivity of solutes are higher when blood flows through circular tube than when it flows between parallel flat plates.
\end{abstract}

\section{Introduction}

The dispersion of a solute in a solvent flowing in a pipe/channel is an important physical phenomenon, which has wide applications in many fields of science and engineering and some potential application fields are chemical engineering, biomedical engineering, physiological fluid dynamics, and environmental sciences [1]. The physics involved in the dispersion theory is the spreading of a passive species (solute) in a flowing fluid (solvent) due to the combined action of molecular diffusion and nonuniform velocity distribution [2]. For better understanding of the concept of shear-augmented dispersion, let us consider a bolus of a solute in the fully developed laminar flow of an incompressible fluid in a conduit. The bolus is carried downstream by the Poiseuille flow and is subjected to the resulting transverse concentration gradient. At the leading edge of the bolus, the bolus diffuses from the high 
concentration region near the centre of the tube towards the low concentration region at the wall [3].

Taylor [4] initiated the study on the dispersion of solutes in fluid flow and reported that if a solute is injected into a solvent flowing steadily in a straight tube, the combined action of the lateral molecular diffusion and the variation of velocity over the cross-section would cause the solute ultimately to spread diffusively with the effective molecular diffusivity $D_{\text {eff }}$ given by $D_{\text {eff }}=a^{2} w_{m}^{2} / 48 D_{m}$, where $D_{m}$ is the molecular diffusivity, $w_{m}$ is the normalized axial velocity, and $a$ is the radius of the tube. He also pointed out that the spreading of the solute is symmetrical about a point moving with the average velocity $w_{m}$ of the fluid. Since many intravenous medications are therapeutic at low concentration, but toxic at high concentration, it is important to know the rate of dispersion of the material in the circulatory system [5]. The main objective of this study is to analyze the dispersion of solutes in blood flow. Sankarasubramanian and Gill [6] discussed the dispersion of solute undergoing firstorder wall retention in Poiseuille flow through circular tube. Their generalized dispersion model gives rise to three effective transport coefficients, namely, the convection, the diffusion, and the exchange coefficients. Lungu and Moffatt [7] analyzed the effect of wall conductance on heat diffusion using Fourier transform with average function to obtain a series solution. Tsangaris and Athanassiadis [8] investigated the diffusion of solutes in an oscillatory flow in an annular pipe.

When blood flows through arteries and veins, it shows many fluid dynamic complexities such as pulsatility, curvature, branching and elasticity of walls, and thus, the dispersion of solutes in blood flow is affected by these factors as well as reactions and the multiphase character of fluid [9]. Hence, it is important to understand the modifications caused by nonNewtonian rheology to the dispersion of passive species. This analysis can also be applied to blood handling devises too. Rao and Deshikachar [10] studied the dispersion of solute in a steady flow of incompressible fluid in an annular pipe and showed that the axial dispersion of the normalized concentration decreases with the increase of the inner radius of the cylinder. They reported that the asymptotic solution, for large time, of effective diffusivity in the flow directions is a decreasing function of the wall conductance. Mazumdar and Das [11] investigated the effect of wall conductance on the axial dispersion in the pulsatile tube flow.

Sharp [12] investigated the shear-augmented dispersion of solutes in the steady flow of Casson fluid through a circular pipe and also flow between parallel plates using Taylor model [4]. Jiang and Grotberg [13] studied the dispersion of a bolus contaminant in a straight tube with oscillatory flow field and weak conductive walls and reported that the axial dispersion diminished by the wall conductance when the frequency parameter exceeds the critical value. Smith and Walton [14] discussed the dispersion of solutes in the fluid flow through inclined tube with an annulus. The dispersion of solutes in the flow of power law fluids was analyzed by Agarwal and Jayaraman [1]. They showed that the effective molecular diffusivity varies with yield stress for Casson and Bingham fluids and power law index in the case of power-law fluids. Dash et al. [15] studied the shear augmented dispersion of a solute in the Casson fluid flow in a conduit using the generalized dispersion model of Gill and Sankarasubramanian [16].

Herschel-Bulkley (H-B) fluid model and Casson fluid model are the non-Newtonian fluid models that are generally used in the studies of blood flow through narrow arteries [17, 18]. Tu and Deville [19] and Sankar et al. [20] mentioned that blood obeys Casson's equation only for moderate shear rate, whereas the H-B equation can still be used at low shear rates and represent fairly closely what is occurring in blood. Several researchers proved that for tube 
diameter $0.095 \mathrm{~mm}$ blood behaves like H-B fluid rather than power law and Bingham fluids [21, 22]. Iida [23] reports "The velocity profile in the arterioles having diameter less than $0.1 \mathrm{~mm}$ are generally explained fairly by Casson and H-B fluid models. However, the velocity profiles in the arterioles whose diameters are less than $0.065 \mathrm{~mm}$ do not conform to the Casson model, but, can still be explained by H-B model." Hence, it is appropriate to model blood as H-B fluid model rather than Casson fluid model when it flows through smaller diameter arteries. The theoretical analysis of shear-augmented dispersion in the steady flow of H-B fluid through circular tube has not been studied so far, to the knowledge of the authors. Hence, in this paper, we analyze the shear augmented dispersion of solutes in the steady flow of $\mathrm{H}-\mathrm{B}$ fluid through a narrow cylindrical tube with possible application to blood flow. Since, some devices involve the flow between parallel flat plates or membranes rather than flow in tubes [12], the study on the dispersion of solutes in fluid flow between parallel flat plates is also important. Thus, it is also aimed to investigate the shear-augmented dispersion of solutes in the incompressible fluid flow between parallel flat plates. The layout of the paper is as follows.

Section 2 formulates the problem mathematically and then solves the resulting system of differential equations to obtain the expression for the flow quantities such as normalized velocity, concentration of the fluid in the core region and outer region, flow rate, and the effective axial diffusivity. The effects of various parameters such as power law index and yield stress on these flow quantities are discussed through appropriate graphs in the numerical simulation of the results and discussion Section 3. Also, some possible physiological application of this study to blood flow is given in Section 3 . The main results are summarized in the concluding Section 4.

\section{Mathematical Formulation}

Consider the dispersion of a solute in the axi-symmetric, steady, laminar, and fully developed unidirectional flow (in the axialdirection) of Herschel-Bulkley (H-B) fluid (viscous incompressiblenon-Newtonian fluid) through (i) circular tube and (ii) between parallel flat plates. The geometry of the flow fields in circular tube and between parallel flat plates are shown in Figures 1(a) and 1(b), respectively.

\subsection{Flow in Circular Tube}

\subsubsection{Governing Equations}

Cylindrical polar coordinate system $(r, \psi, x)$ is used to analyze the flow through uniform circular tube, where $r$ and $x$ are the coordinates in the radial and axial directions, respectively, and $\psi$ is the azimuthal angle. It has been reported that the radial velocity is negligibly

small and can be neglected for a low Reynolds number flow in a narrow artery with mild stenosis [23]. For the steady flow of incompressible viscous fluid, the axial component of the momentum equation simplifies to

$$
\frac{d p}{d x}=-\frac{1}{r} \frac{d}{d r}(r \tau)
$$




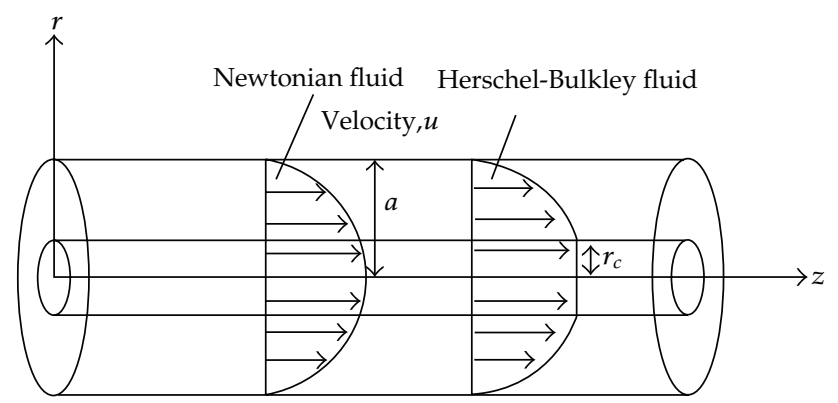

(a) Flow in circular tube

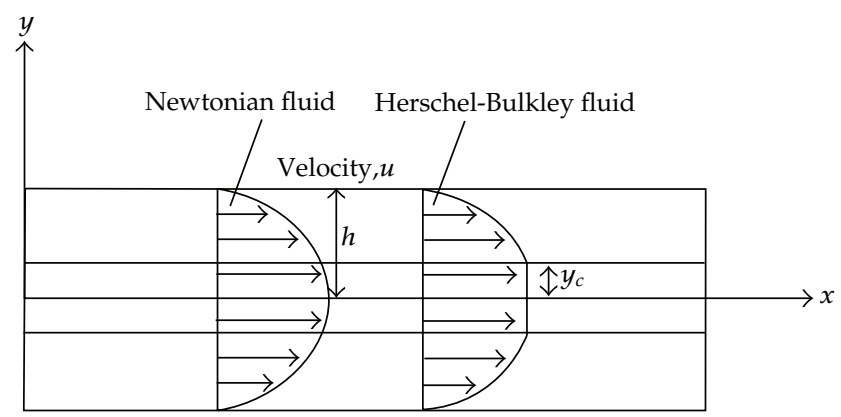

(b) Flow between parallel flat plates

Figure 1: The geometry of the fluid flow.

where $d p / d x$ is the axial pressure gradient, $p$ is the pressure and $\tau$ is the shear stress. The constitutive equation of the H-B fluid is given by

$$
-\frac{d u}{d r}= \begin{cases}\frac{1}{\eta}\left(\tau-\tau_{y}\right)^{n} & \text { if } \tau \geq \tau_{y} \\ 0 & \text { if } \tau \leq \tau_{y}\end{cases}
$$

where $u$ is the velocity in the axial direction; $\eta$ is the coefficient of viscosity of H-B fluid with dimension $\left(M L^{-1} T^{-2}\right)^{n} T$; $\tau_{y}$ is the yield stress and $n$ the is power law index of H-B fluid. To solve (2.1) and (2.2) for the unknowns shear stress $\tau$ and velocity $u$, we utilize the following boundary conditions

$$
\begin{gathered}
\tau \text { is finite at } r=0, \\
u=0 \text { at } r=a,
\end{gathered}
$$

where $a$ is the radius of the tube. For steady flow, the simplified form of the species transport equation in the plug core region and outer (nonplug core) region are given below in (2.5) and (2.6), respectively.

$$
\frac{1}{r} \frac{\partial}{\partial r}\left(r \frac{\partial C_{1}}{\partial r}\right)=\frac{\widehat{u}_{C}}{\mathcal{\kappa}} \frac{\partial C_{1}}{\partial \widetilde{x}}
$$


The species transport equation for the outer region simplifies to the following form:

$$
\frac{1}{r} \frac{\partial}{\partial r}\left(r \frac{\partial C_{2}}{\partial r}\right)=\frac{\widehat{u}}{\mathcal{\kappa}} \frac{\partial C_{2}}{\partial \tilde{x}}
$$

where $C_{1}$ and $C_{2}$ are the concentration of the solute in the plug core region and outer region; $\tilde{x}=x-\bar{u} t$ is the new axial coordinate moving with the normalized velocity $\bar{u} ; \widehat{u}_{C}=u_{C}-\bar{u} ; \widehat{u}=$ $u-\bar{u} ; \widehat{u}$ is the relative velocity in the outer region; $\widehat{u}_{C}$ is the relative velocity in the plug core region; $t$ is the time. The boundary conditions for the concentration of the fluid in the core region are

$$
\begin{aligned}
& \frac{\partial C_{1}}{\partial r}=0 \text { at } r=0, \\
& C_{1}=0 \text { at } r=0, \\
& \frac{\partial C_{2}}{\partial r}=0 \text { at } r=a, \\
& C_{2}=C_{1} \text { at } r=r_{c} .
\end{aligned}
$$

Equations (2.5) and (2.6) can be solved with the help of the boundary conditions (2.7) to get the expressions for the concentrations in the plug core region and outer region.

\subsubsection{Solution Method}

Integrating (2.1) with respect to $r$ and then using (2.3), we get the expression for the shear stress as follows:

$$
\tau=-\frac{r}{2} \frac{d p}{d z}
$$

Using binomial series expansion in (2.2) and neglecting the terms involving $\left(\tau_{y} / \tau\right)^{2}$ and higher powers of $\left(\tau_{y} / \tau\right)$ (since $\left.\left(\tau_{y} / \tau\right)^{3} \ll 1\right)$, one can obtain the simplified form of the constitutive equation as follows:

$$
-\frac{d u}{d r}=\frac{1}{\eta}\left[\tau^{n}-n \tau_{y} \tau^{n-1}+\frac{n(n-1)}{2} \tau_{y} \tau^{n-2}\right]
$$

Using (2.11) in (2.12) and integrating the resulting differential equation with respect to $r$ and then using the boundary condition (2.4), we get the expression for the velocity in the outer (non-plug core) region as

$$
u(r)=\frac{1}{(n+1) \eta}\left(-\frac{1}{2} \frac{d p}{d z}\right)^{n}\left[a^{n+1}-r^{n+1}-(n+1) r_{c}\left(a^{n}-r^{n}\right)+\frac{n(n+1)}{2} r_{c}^{2}\left(a^{n-1}-r^{n-1}\right)\right]
$$


where $r_{c}$ is the plug core radius, which is defined as follows:

$$
r_{c}=\frac{2 \tau_{y}}{-(d p / d z)} .
$$

The expression for the plug flow velocity is obtained by replacing $r$ by $r_{c}$ in (2.13) as given in the following:

$$
u_{c}(r)=\frac{1}{(n+1) \eta}\left(-\frac{1}{2} \frac{d p}{d z}\right)^{n}\left[a^{n+1}-(n+1) r_{c} a^{n}+\frac{n(n+1)}{2} r_{c}^{2} a^{n-1}-\frac{n(n-1)}{2} r_{c}^{n+1}\right] .
$$

Using (2.14) and (2.15), one can obtain the following expression for the bulk velocity or normalized velocity:

$$
\bar{u}=\bar{u}_{H} A\left(z_{c}\right)
$$

where

$$
\begin{gathered}
\bar{u}_{H}=-\frac{a^{n+1}}{\eta(n+3)}\left(-\frac{1}{2} \frac{d p}{d z}\right)^{n} \\
A\left(z_{c}\right)=1-\frac{n(n+3)}{(n+2)} z_{c}+\frac{n(n+3)(n-1)}{2(n+1)} z_{c}^{2}-\frac{\left(n^{4}+2 n^{3}-5 n^{2}-6 n+4\right)}{2(n+1)(n+2)} z_{c}^{n+3}
\end{gathered}
$$

where $z_{c}=r_{c} / a$. When $n=1$, one can get the bulk velocity of Newtonian fluid from (2.17). Solving (2.5) with the help of the boundary conditions (2.7) and (2.8), one can get the expression for the concentration of the solute in the plug core region as follows:

$$
C_{1}=\frac{\bar{u}_{H} r^{2}}{2 \kappa(n+1)}\left(\frac{\partial C_{1}}{\partial \tilde{x}}\right) B\left(z_{c}\right)
$$

where

$$
\begin{aligned}
B\left(z_{c}\right)=1- & \frac{(n+1)(n+3)}{(n+2)} z_{c}+\frac{n(n+3)}{2} z_{c}^{2}-\frac{n(n-1)(n+3)}{4} z_{c}{ }^{n+1} \\
& -\frac{\left(n^{4}+2 n^{3}-5 n^{2}-6 n+4\right)}{2(n+1)(n+2)} z_{c}{ }^{n+3} .
\end{aligned}
$$


One can get the concentration of the solute in the core region by integrating (2.6) and then using the boundary conditions (2.9) and (2.10) which is given as follows:

$$
\begin{aligned}
C_{2}=\frac{\bar{u}_{H} a^{2}}{\kappa}\left(\frac{\partial C_{2}}{\partial \widetilde{x}}\right)[ & -\frac{1}{(n+2)(n+3)} z^{n+3}+\frac{(n+3)}{(n+2)^{2}} z_{c} z^{n+2}-\frac{n(n+3)}{2(n+1)^{2}} z_{c}^{2} z^{n+1} \\
& +z^{2}\left(\frac{1}{2(n+1)}-\frac{(n+3)}{2(n+2)} z_{c}+\frac{n(n+3)}{4(n+1)} z_{c}^{2}\right. \\
& \left.+\frac{\left(n^{4}+2 n^{3}-5 n^{2}-6 n+4\right)}{8(n+1)(n+2)} z_{c}^{n+3}\right) \\
& -\frac{\left(n^{4}+2 n^{3}-5 n^{2}-6 n+4\right)}{4(n+1)(n+2)} z_{c}^{n+3} \log \left(\frac{z}{z_{c}}\right) \\
& \left.-\frac{\left(n^{7}+10 n^{6}+32 n^{5}+18 n^{4}-93 n^{3}-164 n^{2}-52 n+40\right)}{8(n+1)^{2}(n+2)^{2}(n+3)} z_{c}^{n+3}\right] .
\end{aligned}
$$

The flux of solute across a cross section at constant $\tilde{x}$ is defined as follows [12]

$$
q=\frac{1}{\pi a^{2}}\left[\int_{0}^{r_{c}}\left(\widehat{u}_{c} C_{1}-\kappa \frac{\partial C}{\partial \tilde{x}}\right) 2 \pi r d r+\int_{r_{c}}^{a}\left(\widehat{u} C_{2}-\kappa \frac{\partial C}{\partial \tilde{x}}\right) 2 \pi r d r\right] .
$$

For our convenience, (2.22) is rewritten as follows:

$$
q=\left(-\kappa \frac{\partial C}{\partial \tilde{x}}\right)+\frac{2}{a^{2}}\left[I_{1}+I_{2}\right]
$$

where

$$
\begin{gathered}
I_{1}=\int_{0}^{r_{c}} \widehat{u}_{c} C_{1} r d r=\frac{\bar{u}_{H}^{2} B^{2}\left(z_{c}\right) r_{c}^{4}}{4 \kappa(n+1)^{2}}\left(\frac{\partial C}{\partial \widetilde{x}}\right), \\
I_{2}=\int_{r_{c}}^{a}(\widehat{u} r) C_{2} d r=\frac{\bar{u}_{H}^{2} a^{2}}{4 \kappa}\left(\frac{\partial C}{\partial \widetilde{x}}\right) \int_{r_{c}}^{a} T_{1}(r) T_{2}(r) d r .
\end{gathered}
$$


The functions $T_{1}(r)$ and $T_{2}(r)$ appearing in (2.25) are given as follows:

$$
\begin{aligned}
T_{1}= & \frac{(n+3)}{(n+1)} r-\frac{(n+3)}{(n+1)} \frac{r^{n+2}}{a^{n+1}}-(n+3) z_{c} r+(n+3) z_{c} \frac{r^{n+1}}{a^{n}}+\frac{n(n+3)}{2} z_{c}^{2} r \\
& \quad-\frac{n(n+3)}{2} z_{c}^{2} \frac{r^{n}}{a^{n-1}}-A\left(z_{c}\right) r \\
= & T_{11}+T_{12}+T_{13}+T_{14}+T_{15}+T_{16}+T_{17}, \\
T_{2}=- & \frac{1}{(n+1)(n+3)}\left(\frac{r}{a}\right)^{n+3}+\frac{(n+3) z_{c}}{(n+2)^{2}}\left(\frac{r}{a}\right)^{n+2}-\frac{n(n+3)}{2(n+1)^{2}} z_{c}^{2}\left(\frac{r}{a}\right)^{n+1}+G\left(z_{c}\right)\left(\frac{r}{a}\right)^{2} \\
& \quad-\frac{\left(n^{4}+2 n^{3}-5 n^{2}-6 n+4\right)}{4(n+1)(n+2)} z_{c}^{n+3} \log \left(\frac{r}{r_{c}}\right)+H\left(z_{c}\right),
\end{aligned}
$$

where

$$
\begin{gathered}
G\left(z_{c}\right)=\frac{1}{2(n+1)}-\frac{(n+3)}{2(n+2)} z_{c}+\frac{n(n+3)}{4(n+1)} z_{c}^{2}+\frac{\left(n^{4}+2 n^{3}-5 n^{2}-6 n+4\right)}{8(n+1)(n+2)} z_{c}^{n+3}, \\
H\left(z_{c}\right)=\frac{\left(n^{7}+10 n^{6}+32 n^{5}+18 n^{4}-93 n^{3}-164 n^{2}-52 n+40\right)}{8(n+1)^{2}(n+2)(n+3)} z_{c}^{n+3} .
\end{gathered}
$$

For the easy evaluation of the integral in (2.25), it is rewritten as follows:

$$
\begin{aligned}
I_{2} & =\frac{\bar{u}_{H}^{2} a^{2}}{4 \kappa}\left(\frac{\partial C}{\partial \tilde{x}}\right) \int_{r_{c}}^{a} T_{1} T_{2} d r \\
& =\frac{\bar{u}_{H}^{2} a^{2}}{4 \kappa}\left(\frac{\partial C}{\partial \tilde{x}}\right) \int_{r_{c}}^{a}\left(T_{11}+T_{12}+T_{13}+T_{14}+T_{15}+T_{16}+T_{17}\right) T_{2} d r \\
& =\frac{\bar{u}_{H}^{2} a^{2}}{4 \kappa}\left(\frac{\partial C}{\partial \tilde{x}}\right)\left[S_{1}+S_{2}+S_{3}+S_{4}+S_{5}+S_{6}+S_{7}\right]
\end{aligned}
$$

where

$$
\begin{gathered}
S_{1}=\int_{r_{C}}^{a} T_{11} T_{2} d r, \quad S_{2}=\int_{r_{C}}^{a} T_{12} T_{2} d r, \quad S_{3}=\int_{r_{C}}^{a} T_{13} T_{2} d r, \\
S_{4}=\int_{r_{C}}^{a} T_{14} T_{2} d r, \quad S_{5}=\int_{r_{C}}^{a} T_{15} T_{2} d r, \quad S_{6}=\int_{r_{C}}^{a} T_{16} T_{2} d r, \quad S_{7}=\int_{r_{C}}^{a} T_{17} T_{2} d r .
\end{gathered}
$$

The details of obtaining the expressions for $S_{1}, S_{2}, S_{3}, S_{4}, S_{5}, S_{6}$ and $S_{7}$ are given in Appendix A. The effective axial diffusivity is defined as

$$
D_{\text {eff }}=-\frac{q}{\partial C / \partial \tilde{x}}=\kappa\left[1+\frac{\operatorname{Pec}^{2}}{48} \frac{E\left(z_{c}\right)}{A^{2}\left(z_{c}\right)}\right] .
$$


From the simplified form of the expression obtained for the flux of solute (defined in (2.23)), the expression for $E\left(z_{c}\right)$ is obtained as

$$
\begin{aligned}
& E\left(z_{c}\right)=\frac{24}{(n+3)(n+5)}-\frac{48 n(n+3)(2 n+9) z_{c}}{(n+2)(n+4)(n+5)(2 n+5)} \\
& -\frac{12 n\left(n^{6}+13 n^{5}+58 n^{4}+82 n^{3}-91 n^{2}-305 n-142\right) z_{c}^{2}}{(1+n)(2+n)^{3}(4+n)(5+n)} \\
& -\frac{24 n^{2}(n-1)(n+3)(2 n+7) z_{c}^{3}}{(n+1)(n+2)(n+4)(2 n+3)} \\
& -\frac{3(n+3)\left(n^{8}+12 n^{7}+36 n^{6}-46 n^{5}-257 n^{4}+74 n^{3}+404 n^{2}-112 n-64\right) z_{c}^{5+n}}{(n+1)^{2}(n+2)(n+4)(n+5)} \\
& +\frac{24(n+3)^{2}\left(n^{2}+4 n-3\right) z_{c}^{n+6}}{(n+1)(n+2)(n+4)(n+5)} \\
& +\frac{6 n(n+3)\left(n^{4}+6 n^{3}-3 n^{2}-36 n+24\right) z_{c}^{n+7}}{(n+1)(n+4)(n+5)} \\
& -\frac{3\left(\begin{array}{c}
4 n^{13}+48 n^{12}+195 n^{11}+160 n^{10}-913 n^{9}-1878 n^{8}+1865 n^{7}+6772 n^{6} \\
-2535 n^{5}-18918 n^{4}-12512 n^{3}+5656 n^{2}+4872 n-1152
\end{array}\right) z_{c}^{2 n+6}}{(n+1)^{3}(n+2)^{3}(n+3)(2 n+3)(2 n+5)} \\
& +\frac{3\left(n^{4}+2 n^{3}-5 n^{2}-6 n+4\right)\left(n^{4}+6 n^{3}-3 n^{2}-36 n+24\right) z_{c}^{2 n+8}}{(n+1)(n+2)(n+4)(n+5)} \\
& +\frac{6(n-1)(n+3)\left(n^{4}+2 n^{3}-5 n^{2}-6 n+4\right) z_{c}^{n+5} \log \left(z_{c}\right)}{(n+1)(n+2)} \\
& -\frac{6\left(n^{4}+2 n^{3}-5 n^{2}-6 n+4\right)^{2} z_{c}^{2 n+6} \log \left(z_{c}\right)}{(n+1)^{2}(n+2)^{2}} \text {. }
\end{aligned}
$$

\subsection{Flow between Parallel Flat Plates}

\subsubsection{Governing Equations}

Cartesian coordinate system $(x, y)$ is used to analyze the flow between parallel flat plates. The width of the flow region is taken as $2 h$ ( $h$ is half of the spacing between the flat plates). Since, the flow is assumed as steady, laminar, and fully developed, the velocity of the fluid in the $y$ direction is negligibly small and can be neglected for low Reynolds number flow [12]. Thus, for the steady flow of viscous incompressible fluid between the parallel flat plates, the axial component of the momentum equation simplifies to

$$
\frac{d p}{d x}=-\frac{d}{d y}(\tau)
$$


where $\tau$ is the shear stress and $p$ is the pressure. The constitutive equation of the H-B fluid model in Cartesian coordinate system is defined by

$$
-\frac{d u}{d y}= \begin{cases}\frac{1}{\eta}\left(\tau-\tau_{y}\right)^{n} & \text { if } \tau \geq \tau_{y} \\ 0 & \text { if } \tau \leq \tau_{y}\end{cases}
$$

where $u$ is the velocity in the $x$ direction, $\eta$ is the coefficient of viscosity of H-B fluid, $\tau_{y}$ is the yield stress, and $n$ the is power law index of H-B fluid. The following boundary conditions are used to solve (2.32) and (2.33) for the unknowns shear stress $\tau$ and velocity $u$

$$
\begin{gathered}
\tau \text { is finite at } y=0, \\
u=0 \text { at } y=h .
\end{gathered}
$$

The simplified form of the species transport equation in the plug core region and outer region for the flow between flat plates are

$$
\begin{aligned}
& \frac{\partial^{2} C_{1}}{\partial y^{2}}=\frac{\widehat{u}_{c}}{\mathcal{K}} \frac{\partial C_{1}}{\partial \tilde{x}} \\
& \frac{\partial^{2} C_{2}}{\partial y^{2}}=\frac{\widehat{u}}{\mathcal{\kappa}} \frac{\partial C_{2}}{\partial \tilde{x}}
\end{aligned}
$$

where $C_{1}$ and $C_{2}$ are the concentration of the species in the plug core region and outer region, respectively, and $\tilde{x}=x-\bar{u} t$ is the coordinate moving in the $x$ direction with the normalized velocity $\bar{u}, \widehat{u}_{c}=u_{c}-\bar{u}, \widehat{u}=u-\bar{u}, \widehat{u}$ is the relative velocity in the outer region, and $\widehat{u}_{c}$ is the relative velocity in the plug core region, $t$ is the time. The boundary conditions of the concentration of the species in the plug core region and outer region are

$$
\begin{gathered}
\frac{\partial C_{1}}{\partial r}=0 \text { at } y=0, \\
C_{1}=0 \text { at } y=0, \\
\frac{\partial C_{2}}{\partial r}=0 \text { at } y=h, \\
C_{1}=C_{2} \text { at } y=y_{C},
\end{gathered}
$$

where $y_{C}$ is half the width of the plug core region. Equations (2.36) and (2.37) can be solved by utilizing the boundary conditions (2.38)-(2.41) to get the expressions for the concentrations of the solute in the plug core region and outer region. 


\subsubsection{Solution Method}

Integrating (2.32) with respect to $y$ and then using (2.34), one can easily get the following expression for the shear stress $\tau$ :

$$
\tau=-\left(\frac{d p}{d x}\right) y
$$

Using (2.42) in (2.33) and then utilizing the boundary condition (2.35), the expression for the velocity of $\mathrm{H}-\mathrm{B}$ fluid in the outer region is obtained as follows:

$$
u(y)=\frac{1}{(n+1) \eta}\left(-\frac{d p}{d x}\right)^{n}\left[h^{n+1}-y^{n+1}-(n+1) y_{c}\left(h^{n}-y^{n}\right)+\frac{n(n+1)}{2} y_{c}^{2}\left(h^{n-1}-y^{n-1}\right)\right],
$$

where

$$
y_{c}=\frac{\tau_{y}}{-(d p / d x)}
$$

One can obtain the expression for the velocity of $\mathrm{H}-\mathrm{B}$ fluid in the plug core region as below by replacing $y$ by $y_{c}$ in (2.43) and then simplifying the resulting expression

$$
u_{c}(y)=\frac{1}{(n+1) \eta}\left(-\frac{d p}{d x}\right)^{n}\left[h^{n+1}-(n+1) y_{c} h^{n}+\frac{n(n+1)}{2} y_{c}^{2} h^{n-1}-\frac{n(n-1)}{2} y_{c}^{n+1}\right]
$$

The normalized velocity or bulk velocity of the H-B fluid at a cross section is obtained as

$$
\bar{u}=\bar{u}_{H} F\left(z_{c}\right),
$$

where

$$
\begin{gathered}
\bar{u}_{H}=-\frac{h^{n+1}}{(n+2) \eta}\left(-\frac{d p}{d x}\right)^{n}, \\
F\left(z_{c}\right)=1-\frac{n(n+2)}{(n+1)} z_{c}+\frac{(n-1)(n+2)}{2} z_{c}^{2}-\frac{n\left(n^{2}-3\right)}{2(n+1)} z_{c}^{n+2}
\end{gathered}
$$

where $z_{c}=y_{c} / h$. Solving (2.36) with the help of the boundary conditions (2.38) and (2.39), one can get the expression for the concentration of the species in the plug core region as follows:

$$
C_{1}=\frac{\bar{u}_{H} y^{2}}{2 \kappa(n+1)}\left(\frac{\partial C_{1}}{\partial \tilde{x}}\right) M\left(z_{c}\right)
$$


where

$$
M\left(z_{c}\right)=1-(n+2) z_{c}+\frac{(n+1)(n+2)}{2} z_{c}^{2}-\frac{n(n-1)(n+2)}{2} z_{c}{ }^{n+1}+\frac{n\left(n^{2}-3\right)}{2} z_{c}{ }^{n+2} .
$$

The following expression is obtained for the concentration of the species in the outer region by solving (2.37) with the help of the boundary conditions (2.40) and (2.41)

$$
\begin{aligned}
C_{2}=\frac{(n+2) \bar{u}_{H} h^{2}}{\mathcal{K}}\left(\frac{\partial C_{2}}{\partial \tilde{x}}\right)[ & -\frac{1}{(n+1)(n+2)(n+3)} z^{n+3}+\frac{1}{(n+1)(n+2)^{2}} z_{c} z^{n+2}-\frac{1}{2(n+1)^{2}} z_{c}^{2} z^{n+1} \\
& +z^{2}\left(\frac{1}{2(n+1)(n+2)}-\frac{1}{2(n+1)} z_{c}+\frac{1}{4} z_{c}^{2}+\frac{n\left(n^{2}-3\right)}{4(n+1)(n+2)} z_{c}{ }^{n+2}\right) \\
& \left.-\frac{n\left(n^{2}-3\right)}{2(n+1)(n+2)} z_{c}^{n+2} z+\frac{\left(n^{4}+2 n^{3}-5 n^{2}-6 n+4\right)}{4(n+1)(n+2)(n+3)} z_{c}{ }^{n+3}\right],
\end{aligned}
$$

where $z=y / h$. The flux of the solute across a cross section at constant $\tilde{x}$ is defined as

$$
q=\frac{1}{h}\left[\int_{0}^{y_{c}}\left(\widehat{u}_{c} C_{1}-\kappa \frac{\partial C}{\partial \widetilde{x}}\right) d y+\int_{y_{c}}^{h}\left(\widehat{u}_{c} C_{2}-\kappa \frac{\partial C}{\partial \widetilde{x}}\right) d y\right]
$$

For our convenience, (2.51) is rewritten as

$$
q=\left(-\kappa \frac{\partial C}{\partial \tilde{x}}\right)+\frac{1}{h}\left[I_{3}+I_{4}\right]
$$

where

$$
\begin{gathered}
I_{3}=\int_{0}^{y_{C}} \widehat{u}_{c} C_{1}^{\prime} d y=\frac{\bar{u}_{H}^{2} M^{2}\left(z_{c}\right) y_{C}^{3}}{6 \kappa(n+1)^{2}}\left(\frac{\partial C}{\partial \tilde{x}}\right), \\
I_{4}=\int_{y_{C}}^{h} \widehat{u} C_{2} d y=\frac{\bar{u}_{H}^{2}(n+2) h^{2}}{(n+1)^{2} \kappa}\left(\frac{\partial C}{\partial \widetilde{x}}\right) \int_{y_{C}}^{h} W_{1}(y) W_{2}(y) d y .
\end{gathered}
$$


The functions $W_{1}(y)$ and $W_{2}(y)$ appearing in (2.54) are

$$
\begin{aligned}
W_{1}(y)= & \frac{1}{(n+1)}-\frac{(n+2)}{(n+1)} \frac{y^{n+1}}{h^{n+1}}-\frac{(n+2)}{(n+1)} z_{c}+(n+2) z_{c} \frac{y^{n}}{h^{n}}+\frac{(n+2)}{2} z_{c}^{2} \\
& -\frac{n(n+2)}{2} z_{c}^{2} \frac{y^{n-1}}{h^{n-1}}-\frac{n\left(n^{2}-3\right)}{2(n+1)} z_{c}^{n+2} \\
= & W_{11}+W_{12}+W_{13}+W_{14}+W_{15}+W_{16}+W_{17}, \\
W_{2}(y)=- & \frac{1}{(n+1)(n+2)(n+3)}\left(\frac{y}{h}\right)^{n+3}+\frac{z_{c}}{(n+1)(n+2)}\left(\frac{y}{h}\right)^{n+2}-\frac{1}{2(n+1)} z_{c}^{2}\left(\frac{y}{h}\right)^{n+1} \\
& +J\left(z_{c}\right)\left(\frac{y}{h}\right)^{2}-\frac{n\left(n^{2}-3\right)}{2(n+1)(n+2)} z_{c}^{n+2}\left(\frac{y}{h}\right)+K\left(z_{c}\right),
\end{aligned}
$$

where

$$
\begin{gathered}
J\left(z_{c}\right)=\frac{1}{2(n+1)(n+2)}-\frac{1}{2(n+1)} z_{c}+\frac{1}{4} z_{c}^{2}+\frac{n\left(n^{2}-3\right)}{4(n+1)(n+2)} z_{c}^{n+2}, \\
K\left(z_{c}\right)=\frac{\left(n^{4}+2 n^{3}-5 n^{2}-6 n+4\right)}{4(n+1)(n+2)(n+3)} z_{c}^{n+3} .
\end{gathered}
$$

Equation (2.54) is rewritten as below for the easy evaluation of the integral appearing in it

$$
\begin{aligned}
I_{4} & =\frac{(n+2) \bar{u}_{H}^{2} h^{2}}{\kappa}\left(\frac{\partial C}{\partial \tilde{x}}\right) \int_{y_{C}}^{h} W_{1}(y) W_{2}(y) d y \\
& =\frac{(n+2) \bar{u}_{H}^{2} h^{2}}{\kappa}\left(\frac{\partial C}{\partial \tilde{x}}\right) \int_{y_{C}}^{h}\left(W_{11}+W_{12}+W_{13}+W_{14}+W_{15}+W_{16}+W_{17}\right) W_{2} d y \\
& =\frac{\bar{u}_{H}^{2} a^{2}}{4 \kappa}\left(\frac{\partial C}{\partial \tilde{x}}\right)\left[D_{1}+D_{2}+D_{3}+D_{4}+D_{5}+D_{6}+D_{7}\right]
\end{aligned}
$$

where

$$
\begin{gathered}
D_{1}=\int_{y_{c}}^{h} W_{11} W_{2} d y, \quad D_{2}=\int_{y_{c}}^{h} W_{12} W_{2} d y, \quad D_{3}=\int_{y_{c}}^{h} W_{13} W_{2} d y, \\
D_{4}=\int_{y_{c}}^{h} W_{14} W_{2} d y, \quad D_{5}=\int_{y_{c}}^{h} W_{15} W_{2} d y, \\
D_{6}=\int_{y_{C}}^{h} W_{16} W_{2} d y, \quad D_{7}=\int_{y_{C}}^{h} W_{17} W_{2} d y .
\end{gathered}
$$


The details of obtaining the expressions for $D_{1}, D_{2}, D_{3}, D_{4}, D_{5}, D_{6}$ and $D_{7}$ are given in Appendix B. The effective axial diffusivity is defined as

$$
D_{\mathrm{eff}}=-\frac{q}{\partial C / \partial \tilde{x}}=\kappa\left[1+\frac{2 \mathrm{Pec}^{2}}{105} \frac{N\left(z_{c}\right)}{F^{2}\left(z_{c}\right)}\right]
$$

where

$$
\begin{aligned}
& N\left(z_{c}\right)=\frac{35}{(n+4)(2 n+5)}-\frac{35 n(2 n+7) z_{c}}{2(n+1)(n+3)(n+4)} \\
&+ \frac{35\left(n^{4}+6 n^{3}+8 n^{2}-3 n-3\right) z_{c}^{2}}{(n+1)^{2}(n+3)(n+4)}-\frac{35 n(n-1)(n+2) z_{c}^{3}}{8(n+1)(n+3)} \\
&+\frac{35(n-1)^{2}(n+2) z_{c}^{4}}{4(2 n+1)}-\frac{35 n(n+6)\left(n^{2}-3\right) z_{c}^{n+2}}{4(n+1)(n+3)(n+4)} \\
&+\frac{35 n^{2}(n+5)\left(n^{2}-3\right) z_{c}^{n+3}}{4(n+1)^{2}(n+3)} \\
&-\frac{35\left(n^{7}+10 n^{6}+24 n^{5}-32 n^{4}-133 n^{3}+22 n^{2}+156 n-24\right) z_{c}^{n+4}}{8(n+1)^{2}(n+3)(n+4)} \\
&-\frac{35(n+2)\left(n^{4}+4 n^{3}-5 n^{2}-18 n+12\right) z_{c}^{n+5}}{4(n+1)(n+3)(n+4)} \\
&+\frac{35(n+2)\left(n^{4}+4 n^{3}-5 n^{2}-18 n+12\right) z_{c}^{n+6}}{8(n+3)(n+4)}+\frac{35 n^{2}\left(n^{2}-3\right)^{2} z_{c}^{2 n+4}}{8(n+1)^{2}} \\
&-\frac{35\left(957 n^{10}+16 n^{9}+80 n^{8}-4 n^{7}-492 n^{6}-380 n^{5}-113 n^{4}+740 n^{3}-396 n^{2}-192 n+72\right) z_{c}^{2 n+5}}{16(n+1)^{2}(n+3)(2 n+1)(2 n+5)} \\
&+\frac{35 n\left(n^{2}-3\right)\left(n^{4}+4 n^{3}-5 n^{2}-18 n+12\right) z_{c}^{2 n+6}}{8(n+1)(n+3)(n+4)} . \\
& \\
& \\
& \\
&
\end{aligned}
$$

\section{Results and Discussion}

The objective of this study is to analyze the blood flow characteristics due to the shear augmented dispersion of solutes when blood flows (i) through circular tubes and (ii) between parallel flat plates, modeling blood as H-B fluid. It is also aimed to discuss the effects of various physical parameters on the velocity distribution of blood, relative diffusivity, and effective axial diffusivity of the solute [19]. 


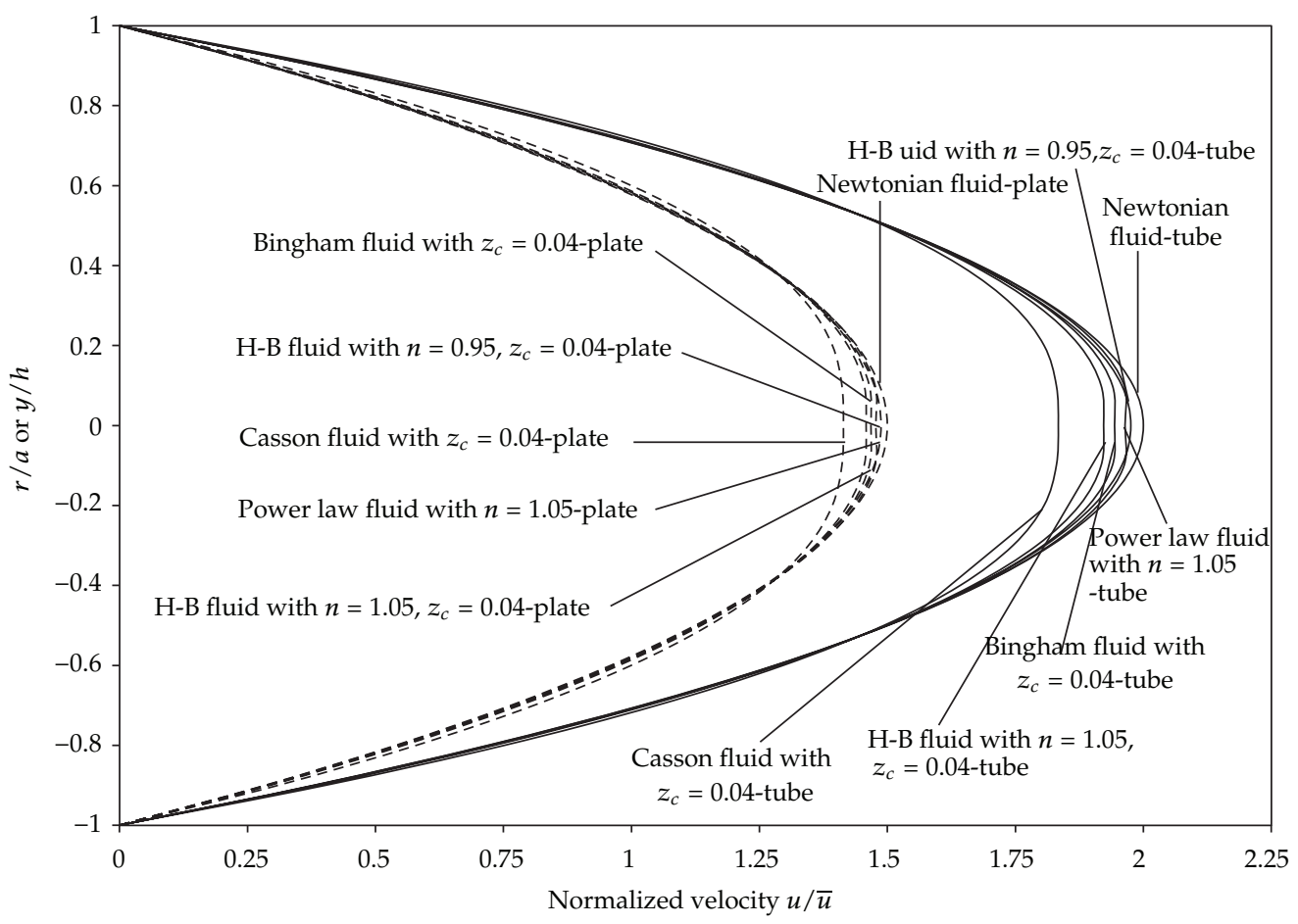

Figure 2: Normalized velocity profiles of some non-Newtonian fluids flowing in a tube and between parallel flat plates.

\subsection{Normalized Velocity Distribution}

The normalized velocity profiles of H-B and Casson fluids (for different values of the power law index $z_{c}$ and yield stress $n$ ) flow (i) through circular tube and (ii) between parallel flat plates are sketched in Figure 2. It is observed that the normalized velocity decreases marginally with the increase of power law index and yield stress. It is also seen that the normalized velocity of Newtonian fluid model is marginally higher than those of the H-B and Casson fluid models, and it is slightly higher than that of Power law fluid model. It is clear that the normalized velocity of H-B fluid model is considerably higher than that of the Casson fluid model. One can notice that the normalized velocity of any fluid model when it flows between parallel flat plates is very similar to its normalized velocity when it flows through a circular tube. It is of interest to note that the normalized velocity profile of the Newtonian fluid model is in good agreement with the corresponding normalized velocity profile in Figure 3 of Sharp [12].

\subsection{Relative Diffusivity}

The variation of relative diffusivity with yield stress of $\mathrm{H}-\mathrm{B}$ and Casson fluids when flowing (i) through circular tube and (ii) between parallel flat plates is shown in Figure 3. It is observed that the relative diffusivity decreases slowly with the increase of the yield stress of $\mathrm{H}-\mathrm{B}$ and Bingham fluid models, but it decreases rapidly (nonlinearly) with the increase of the yield stress for Casson fluid model. It is also noted that the relative diffusivity decreases 


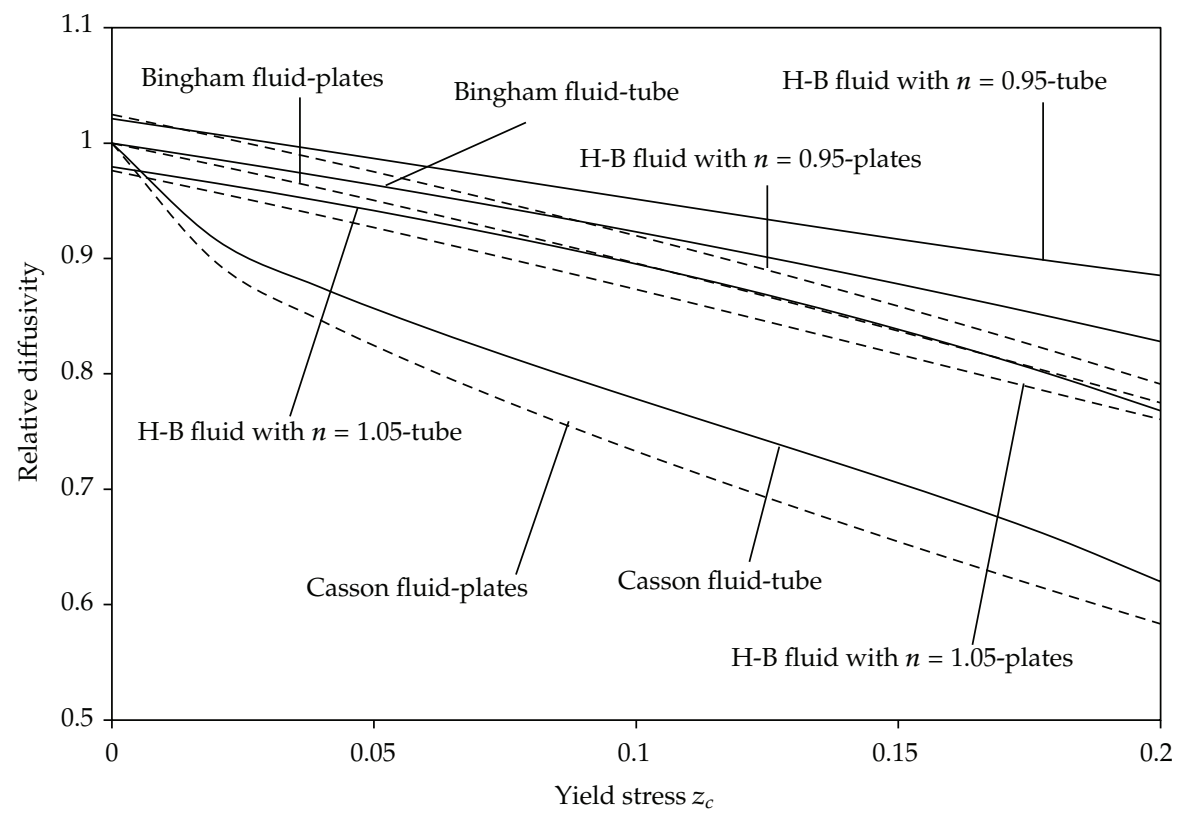

Figure 3: Variation of the relative diffusivity with yield stress for some non-Newtonian fluids when they flow in a tube and between parallel flat plates.

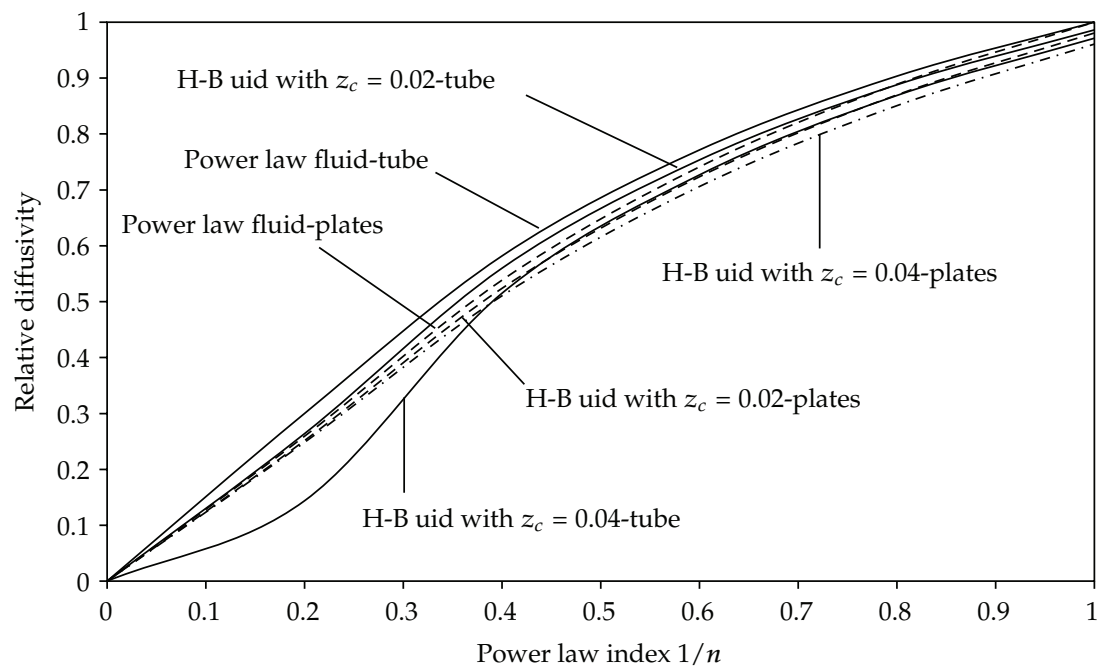

Figure 4: Comparison of relative diffusivity of non-Newtonian fluids when flowing in a tube and between parallel flat plates.

considerably with the increase of the power law index of the H-B fluid model. It is also found that the relative diffusivity is significantly higher for $\mathrm{H}-\mathrm{B}$ fluid model than that of the Casson fluid model. It is seen that for any fluid model, the relative diffusivity is higher when it flows through circular pipe than when it flows between parallel flat plates.

Figure 4 sketches the variation of relative diffusivity with the reciprocal of the power law index for H-B and power law fluids when they flow (i) through a circular tube and 
Table 1: Estimates of yield stress $z_{c}$ and relative diffusivity $\left(E / A^{2}\right)$ in the canine vascular system for flow in tube.

\begin{tabular}{lccccccc}
\hline & \multicolumn{1}{l}{$\begin{array}{c}\text { Velocity } \\
(\mathrm{cm} / \mathrm{s})\end{array}$} & $\begin{array}{c}\text { Diameter } \\
(\mathrm{cm})\end{array}$ & $z_{c}$ & \multicolumn{2}{c}{ H-B fluid } & Bingham fluid Casson fluid \\
& & & & $n=0.95$ & $n=1.05$ & & \\
\hline Arterioles & 0.75 & 0.005 & 0.00654 & 1.0186 & 0.9749 & 0.9955 & 0.9544 \\
Venules & 0.35 & 0.004 & 0.01120 & 1.0141 & 0.9716 & 0.9922 & 0.9391 \\
Inferior vena cava & 25.00 & 1.000 & 0.03920 & 0.9863 & 0.9503 & 0.9719 & 0.8761 \\
Ascending aorta & 20.00 & 1.500 & 0.07370 & 0.9495 & 0.9207 & 0.9450 & 0.8179 \\
\hline
\end{tabular}

Table 2: Estimates of yield stress $z_{c}$ and relative diffusivity $\left(N / F^{2}\right)$ in the canine vascular system for flow between parallel flat plates.

\begin{tabular}{lccccccc}
\hline & \multicolumn{1}{l}{$\begin{array}{c}\text { Velocity } \\
(\mathrm{cm} / \mathrm{s})\end{array}$} & $\begin{array}{c}\text { Diameter } \\
(\mathrm{cm})\end{array}$ & $z_{c}$ & H-B fluid & Bingham fluid & Casson fluid \\
& & & & $n=0.95$ & $n=1.05$ & & 0.9434 \\
\hline Arterioles & 0.75 & 0.005 & 0.00654 & 1.0167 & 0.9700 & 0.9938 & 0.9245 \\
Venules & 0.35 & 0.004 & 0.01120 & 1.0136 & 0.9655 & 0.9894 & 0.8479 \\
Inferior vena cava & 25.00 & 1.000 & 0.03920 & 0.9942 & 0.9378 & 0.9616 & 0.7787 \\
Ascending aorta & 20.00 & 1.500 & 0.07370 & 0.9700 & 0.9018 & 0.9251 & \\
\hline
\end{tabular}

(ii) between flat plates. It is clear that for power law fluid, the relative diffusivity increases rapidly with the increase of the reciprocal of the power law index from 0 to 0.5 , and then it increases slowly with the increase of the reciprocal of the power law index from 0.5 to 1 . The same behavior is also noticed for H-B fluid, but some nonlinearity is found at lower values of the reciprocal of the power law index. It is noted that for both H-B and power law fluid models, the relative diffusivity increases almost linearly with the increase of the reciprocal of the power law index. It is found that the relative diffusivity is marginally higher for power law fluid model than that of the H-B fluid model.

\subsection{Some Physiological Applications}

The estimates of yield stress $z_{c}$ and relative diffusivity in the canine vascular system are useful to understand the dispersion of solutes in blood flow through arterioles, venules, inferior vena cava, and ascending aorta. Using the expressions obtained for flow in tubes, the estimates of yield stress $z_{c}$ and relative diffusivity in the canine vascular system $[12,24]$ (in arteries of different diameters) are computed in Table 1. It is observed that the estimates of the relative diffusivity decreases slowly with the increase of the yield stress. It is also noted that the relative diffusivity decreases gradually with the increase of the power law index. It is found that the solute disperses rapidly in arterioles than in ascending aorta. It is also noticed that the solute dispersion is faster when blood is modeled by H-B fluid or Bingham fluid than when it is modeled by Casson fluid.

From the expressions obtained for flow between parallel flat plates, the estimates of yield stress $z_{c}$ and relative diffusivity in the same canine vascular system are computed in Table 2 . It is noted that the variation in the relative diffusivity with the yield stress/diameter 
of the canine artery is the similar to the one that was observed in the case of flow in tube flow in Table 1. From Tables 1 and 2, it is observed that for any fluid model, the relative diffusivity of the solute is slightly higher when it disperses in circular tube than when it disperses between parallel flat plates.

\section{Conclusion}

This mathematical analysis exhibits many interesting results on the dispersion of the solutes in blood flow when blood is modeled by H-B fluid model and compares the results of the present study with the results of Sharp [12]. The main findings of this theoretical study are summarized as follows.

(i) The normalized velocity of blood flow is considerably higher when it is modeled by H-B fluid rather than Casson fluid model.

(ii) The normalized velocity of blood is significantly higher when it flows through circular tube than when it flows between parallel plates.

(iii) The relative diffusivity and axial diffusivity of the solute are marginally higher when blood is modeled by H-B fluid rather than by Casson fluid.

(iv) The relative diffusivity and axial diffusivity of the solute are slightly higher when blood flows in circular tubes than when it flows between parallel flat plates.

(v) The normalized velocity of blood, relative diffusivity, and axial diffusivity of solute decrease with the increase of the yield stress of the blood.

Based on these results, one can note that there is a substantial difference between the flow quantities of H-B fluid model (present results) and Casson fluid model (results of Sharp [12]), and thus, it is expected that the present H-B model may be useful to predict physiologically important flow quantities. Hence, it is concluded that the present study can be treated as an improvement in the mathematical modeling of dispersion of solutes in blood flow through narrow diameter arteries. Since the solutes may disperse unsteadily, the study on the unsteady diffusion of solutes in blood flow with effects on boundary absorption would be more realistic, and this will be done in the near future.

\section{Appendices}

A.

$$
\begin{gathered}
S_{1}=a^{2}\left[\frac{(n+7)}{8(n+1)(n+5)}-\frac{\left(n^{4}+12 n^{3}+45 n^{2}+54 n\right) z_{c}}{8(n+1)(n+2)^{2}(n+4)}+\frac{\left(n^{4}+7 n^{3}+7 n^{2}-15 n\right) z_{c}^{2}}{16(n+1)^{3}}\right. \\
-\frac{(n+3) z_{c}^{4}}{8(n+1)^{2}}+\frac{(n+3)^{2}}{8\left(n^{2}+3 n+2\right)} z_{c}^{5}-\frac{n(n+3)^{2}}{16(n+1)^{2}} z_{c}^{6}
\end{gathered}
$$


Journal of Applied Mathematics

$$
\begin{aligned}
& +\frac{\left(n^{7}+4 n^{6}-10 n^{5}-60 n^{4}-39 n^{3}+112 n^{2}+128 n-8\right)}{32(n+1)^{3}(n+2)^{2}} z_{c}^{n+3} \\
& -\frac{\left(n^{5}+11 n^{4}+37 n^{3}+27 n^{2}-42 n-18\right)}{8(n+1)^{2}(n+4)(n+5)} z_{c}^{n+5}-\frac{(n+3)\left(n^{4}+2 n^{3}-5 n^{2}-6 n+4\right)}{32(n+1)^{2}(n+2)} z_{c}^{n+7} \\
& \left.+\frac{(n+3)\left(n^{4}+2 n^{3}-5 n^{2}-6 n+4\right)}{8(n+1)^{2}(n+2)} z_{c}^{n+3} \log z_{c}\right] \\
& S_{2}=a^{2}\left[-\frac{(n+4)}{2(n+1)(n+3)(n+5)}+\frac{n\left(2 n^{3}+19 n^{2}+60 n+63\right)}{2(n+1)(n+2)^{2}(n+5)(2 n+5)} z_{c}\right. \\
& +\frac{n\left(n^{4}+8 n^{3}+18 n^{2}-27\right)}{4(n+1)^{3}(n+2)(n+5)} z_{c}^{2}+\frac{\left(n^{6}+7 n^{5}+17 n^{4}-n^{3}-54 n^{2}-50 n+8\right) z_{c}^{n+3}}{4(n+1)^{3}(n+2)^{2}(n+5)} \\
& +\frac{(n+3) z_{c}^{n+5}}{2(n+1)^{2}(n+5)}-\frac{(n+3)^{2} z_{c}^{n+6}}{2\left(n^{3}+8 n^{2}+17 n+10\right)}+\frac{n(n+3)^{2}}{4(n+1)^{2}(n+5)} z_{c}^{n+7} \\
& -\frac{\left(2 n^{6}+15 n^{5}+39 n^{4}+33 n^{3}-25 n^{2}-52 n-8\right)}{8(n+1)^{2}(n+2)(n+3)(2 n+5)} z_{c}^{2 n+6} \\
& \left.+\frac{(n+3)\left(n^{4}+2 n^{3}-5 n^{2}-6 n+4\right)}{8(n+1)^{2}(n+2)(n+5)} z_{c}^{2 n+8}-\frac{\left(n^{4}+2 n^{3}-5 n^{2}-6 n+4\right)}{4(n+1)^{2}(n+2)} z_{c}^{n+3} \log z_{c}\right], \\
& S_{3}=a^{2}\left[-\frac{n(n-1)(n+3)(n+5)}{16(n+1)^{2}}-\frac{(n+7)}{8(n+5)} z_{c}+\frac{n(n+3)^{2}(n+6)}{8(n+2)^{2}(n+4)} z_{c}^{2}+\frac{(n+3)}{8(n+1)} z_{c}^{5}\right. \\
& -\frac{(n+3)^{2}}{8(n+2)} z_{c}^{6}+\frac{n(n+3)^{2}}{16(n+1)} z_{c}^{7}-\frac{\left(n^{7}+4 n^{6}-10 n^{5}-60 n^{4}-39 n^{3}+112 n^{2}+128 n-8\right)}{32(n+1)^{2}(n+2)^{2}} z_{c}^{n+4} \\
& +\frac{(n+3)\left(n^{4}+8 n^{3}+13 n^{2}-12 n-6\right)}{8(n+1)(n+4)(n+5)} z_{c}^{n+6} \\
& +\frac{(n+3)\left(n^{4}+2 n^{3}-5 n^{2}-6 n+4\right)}{32(n+1)(n+2)} z_{c}^{n+8} \\
& \left.-\frac{(n+3)\left(n^{4}+2 n^{3}-5 n^{2}-6 n+4\right)}{8(n+1)(n+2)} z_{c}^{n+4} \log z_{c}\right] \\
& S_{4}=a^{2}\left[\frac{(2 n+7)}{2(n+4)(2 n+5)} z_{c}-\frac{n(n+3)^{3}}{2(n+2)^{3}(4+n)} z_{c}^{2}+\frac{n(n-1)(n+3)^{2}(2 n+5)}{4(n+1)^{2}(n+4)(2 n+3)} z_{c}^{3}\right. \\
& -\frac{\left(n^{6}+7 n^{5}+15 n^{4}+n^{3}-44 n^{2}-56 n+4\right)}{4(n+1)^{2}(n+2)^{2}(n+4)} z_{c}^{n+4}-\frac{(n+3)}{2(n+1)(n+4)} z_{c}^{n+5} \\
& +\frac{(n+3)}{4(n+2)^{2}(n+4)} z_{c}^{n+6}-\frac{n(n+3)^{2}}{2(n+2)} z_{c}^{n+7}
\end{aligned}
$$




$$
\begin{aligned}
& +\frac{(n+3)^{2}\left(4 n^{6}+28 n^{5}+51 n^{4}-15 n^{3}-97 n^{2}-39 n+24\right)}{8(n+1)(n+2)^{3}(2 n+3)(2 n+5)} z_{c}^{2 n+6} \\
& -\frac{(n+3)\left(n^{4}+2 n^{3}-5 n^{2}-6 n+4\right)}{8(n+1)(n+2)(n+4)} z_{c}^{2 n+8} \\
& \left.+\frac{(n+3)\left(n^{4}+2 n^{3}-5 n^{2}-6 n+4\right)}{4(n+1)(n+2)^{2}} z_{c}^{n+4} \log z_{c}\right], \\
& S_{5}=a^{2}\left[\frac{n(n+7)}{16(n+5)} z_{c}^{2}-\frac{n^{2}(n+3)^{2}(n+6)}{16(n+2)^{2}(n+4)} z_{c}^{3}+\frac{n^{2}(n-1)(n+3)(n+5)}{32(n+1)^{2}} z_{c}^{4}\right. \\
& -\frac{n(n+3)}{16(n+1)} z_{c}^{6}+\frac{n(n+3)^{2}}{16(n+2)} z_{c}^{7}-\frac{n^{2}(n+3)^{2}}{32(n+1)} z_{c}^{8} \\
& -\frac{n\left(n^{7}+4 n^{6}-10 n^{5}-60 n^{4}-39 n^{3}+112 n^{2}+128 n-8\right)}{64(n+1)^{2}(n+2)^{2}} z_{c}^{n+5} \\
& +\frac{n(n+3)\left(n^{4}+8 n^{3}+13 n^{2}-12 n-6\right)}{16(n+1)(n+4)(n+5)} z_{c}^{n+7}-\frac{n(n+3)\left(n^{4}+2 n^{3}-5 n^{2}-6 n+4\right)}{64(n+1)(n+2)} z_{c}^{n+9} \\
& \left.+\frac{(n+3)\left(n^{4}+2 n^{3}-5 n^{2}-6 n+4\right)}{16(n+1)(n+2)} z_{c}^{n+5} \log z_{c}\right] \\
& S_{6}=a^{2}\left[-\frac{n}{4(n+2)} z_{c}^{2}+\frac{n^{2}(n+3)(2 n+5)}{4(n+2)^{2}(2 n+3)} z_{c}^{3}-\frac{n^{2}(n-1)(n+2)(n+3)}{8(n+1)^{3}} z_{c}^{4}\right. \\
& +\frac{n^{2}\left(n^{4}+4 n^{3}+3 n^{2}+2 n+10\right)}{8(n+1)^{2}(n+2)^{2}} z_{c}^{n+5}-\frac{n(n+3)}{4(n+2)} z_{c}^{n+6}+\frac{n^{2}(n+3)}{8(n+1)} z_{c}^{n+7} \\
& -\frac{n(n+3)^{2}\left(2 n^{5}+3 n^{4}-2 n^{3}+n^{2}+2 n-4\right)}{16(n+1)^{3}(n+2)(2 n+3)} z_{c}^{2 n+6} \\
& \left.+\frac{n\left(n^{4}+2 n^{3}-5 n^{2}-6 n+4\right)}{16(n+1)(n+2)} z_{c}^{2 n+8}-\frac{n(n+3)\left(n^{4}+2 n^{3}-5 n^{2}-6 n+4\right)}{8(n+1)^{2}(n+2)} z_{c}^{n+5} \log z_{c}\right], \\
& S_{7}=-a^{2}\left[\frac{(n+7)}{8(n+3)(n+5)}+\frac{n\left(2 n^{3}+27 n^{2}+113 n+146\right)}{8(n+2)^{2}(n+4)^{2}(n+5)} z_{c}\right. \\
& -\frac{n\left(n^{7}+19 n^{6}+142 n^{5}+538 n^{4}+1078 n^{3}+1005 n^{2}+147 n-242\right)}{8(n+1)^{2}(n+2)^{3}(n+4)(n+5)} z_{c}^{2} \\
& +\frac{\left(n^{6}+6 n^{5}-22 n^{3}+11 n^{2}-8 n-4\right)}{32(n+1)^{3}} z_{c}^{4}-\frac{(n+3)(2 n+1)}{8(n+1)(n+2)} z_{c}^{5} \\
& +\frac{n(n+3)(2 n+3)\left(n^{2}+3 n+1\right)}{8(n+1)^{2}(n+2)^{2}} z_{c}^{6}+\frac{n(n+3)(2 n-1)}{16(n+1)(n+2)} z_{c}^{7}
\end{aligned}
$$


Journal of Applied Mathematics

$$
\begin{aligned}
& -\frac{\left(n^{8}+7 n^{7}-14 n^{6}-186 n^{5}-347 n^{4}+203 n^{3}+1024 n^{2}+616 n-152\right)}{32(n+1)^{2}(n+2)^{2}(n+3)(n+5)} z_{c}^{n+3} \\
& +\frac{n\left(n^{8}+6 n^{7}-18 n^{6}-184 n^{5}-311 n^{4}+266 n^{3}+1000 n^{2}+504 n-176\right)}{32(n+1)^{2}(n+2)^{3}(n+4)} z_{c}^{n+4} \\
& +\frac{n\left(n^{10}+21 n^{9}+150 n^{8}+378 n^{7}-235 n^{6}-2391 n^{5}-2388 n^{4}+1288 n^{3}+1800 n^{2}+608 n+192\right)}{64(n+1)^{2}(n+2)^{2}(n+4)(n+5)} z_{c}^{n+5} \\
& +\frac{(n+3)\left(n^{4}+8 n^{3}+13 n^{2}-12 n-6\right)}{8(n+1)(n+2)(n+4)(n+5)} z_{c}^{n+6} \\
& -\frac{(n-1)\left(2 n^{7}+25 n^{6}+107 n^{5}+169 n^{4}+35 n^{3}-54 n^{2}+12 n-80\right)}{32(n+1)^{2}(n+2)(n+4)(n+5)} z_{c}^{n+7} \\
& -\frac{(n-2)(n+3)\left(n^{4}+2 n^{3}-5 n^{2}-6 n+4\right)}{32(n+1)(n+2)^{2}} z_{c}^{n+8} \\
& +\frac{n(n-3)(n+3)\left(n^{4}+2 n^{3}-5 n^{2}-6 n+4\right)}{64(n+1)^{2}(n+2)} z_{c}^{n+9} \\
& +\frac{\left(n^{4}+2 n^{3}-5 n^{2}-6 n+4\right)\left(n^{7}+4 n^{6}-10 n^{5}-60 n^{4}-39 n^{3}+112 n^{2}+128 n-8\right)}{64(n+1)^{2}(n+2)^{3}(n+3)} z_{c}^{2 n+6} \\
& -\frac{\left(n^{4}+2 n^{3}-5 n^{2}-6 n+4\right)\left(n^{4}+8 n^{3}+13 n^{2}-12 n-6\right)}{16(n+1)^{2}(n+2)(n+4)(n+5)} z_{c}^{2 n+8} \\
& -\frac{\left(n^{4}+2 n^{3}-5 n^{2}-6 n+4\right)^{2}}{64(n+1)^{2}(n+2)^{2}} z_{c}^{2 n+10}-\frac{\left(n^{4}+2 n^{3}-5 n^{2}-6 n+4\right)}{8(n+1)(n+2)} z_{c}^{n+3} \log \left(z_{c}\right) \\
& +\frac{n(n+3)\left(n^{4}+2 n^{3}-5 n^{2}-6 n+4\right)}{8(n+1)(n+2)^{2}} z_{c}^{n+4} \log \left(z_{c}\right) \\
& -\frac{n(n-1)(n+3)\left(n^{4}+2 n^{3}-5 n^{2}-6 n+4\right)}{16(n+1)^{2}(n+2)} z_{c}^{n+5} \log \left(z_{c}\right) \\
& \left.+\frac{\left(n^{4}+2 n^{3}-5 n^{2}-6 n+4\right)}{16(n+1)^{2}(n+2)^{2}} z_{c}^{2 n+6} \log \left(z_{c}\right)\right] \text {. }
\end{aligned}
$$

B.

$$
\begin{gathered}
W_{1}=h\left[\frac{(n+6)}{6(n+1)(n+2)(n+3)(n+4)}-\frac{n(n+5) z_{c}}{6(n+1)^{2}(n+2)(n+3)}+\frac{(n-1)(n+4) z_{c}^{2}}{12(n+1)^{2}(n+2)}\right. \\
-\frac{z_{c}^{3}}{6(n+1)^{2}(n+2)}+\frac{z_{c}^{4}}{6(n+1)^{2}}+\frac{1}{12(n+1)} z_{c}^{5}
\end{gathered}
$$




$$
\begin{aligned}
& -\frac{n\left(n^{2}-3\right)^{2}}{6(n+1)^{2}(n+2)} z_{c}^{n+2}+\frac{\left(n^{4}+2 n^{3}+112 n^{2}-n-8\right)}{4(n+1)^{2}(n+2)(n+3)} z_{c}^{n+3} \\
& \left.+\frac{\left(n^{4}+6 n^{3}+7 n^{2}-6 n-4\right)}{4(n+1)^{2}(n+2)(n+3)(n+4)} z_{c}^{n+4}-\frac{n\left(n^{2}-3\right)}{12(n+1)^{2}(n+2)} z_{c}^{n+5}\right] \\
& W_{2}=h\left[-\frac{(2 n+7)}{2(n+1)(n+3)(n+4)(2 n+5)}+\frac{n(n+3)}{2(n+1)^{2}(n+2)(n+4)} z_{c}\right. \\
& \left.+\frac{(n-1)(n+2)(2 n+5)}{4(n+1)^{2}(n+4)(2 n+3)} z_{c}^{2}+\frac{n\left(n^{2}-3\right) z_{c}^{2 n+6}}{4(n+1)^{2}(n+4)}\right], \\
& W_{3}=h\left[-\frac{(n+6)}{6(n+1)(n+3)(n+4)} z_{c}+\frac{n(n+5)}{6(n+1)^{2}(n+3)} z_{c}^{2}-\frac{(n-1)(n+4)}{12(n+1)^{2}} z_{c}^{3}\right. \\
& +\frac{1}{6(n+1)^{2}} z_{c}^{4}+\frac{(n+2)}{12(n+1)} z_{c}^{6}+\frac{n\left(n^{2}-3\right)}{6(n+1)^{2}} z_{c}^{n+3} \\
& -\frac{\left(n^{4}+2 n^{3}-5 n^{2}-6 n+4\right)}{4(n+1)^{2}(n+3)} z_{c}^{n+4}-\frac{\left(n^{4}+6 n^{3}+7 n^{2}-6 n-4\right)}{4(n+1)^{2}(n+3)(n+4)} z_{c}^{n+5} \\
& \left.+\frac{n\left(n^{2}-3\right)}{12(n+1)^{2}} z_{c}^{n+6}\right] \\
& W_{4}=h\left[\frac{1}{2(n+2)(n+3)} z_{c}-\frac{n(2 n+5)}{2(n+1)(n+3)(2 n+3)} z_{c}^{2}+\frac{(n-1)(n+2)^{2}}{4(n+1)^{2}(n+3)} z_{c}^{3}\right. \\
& -\frac{n(n+4)\left(n^{2}-3\right)}{4(n+1)(n+2)(n+3)} z_{c}^{n+3}+\frac{\left(n^{4}+2 n^{3}-5 n^{2}-8 n+2\right)}{4(n+1)^{2}(n+3)} z_{c}^{n+4} \\
& +\frac{(n+2)}{2(n+1)(n+3)} z_{c}^{n+5}-\frac{(n+2)}{4(n+3)} z_{c}^{n+6} \\
& \left.+\frac{\left(2 n^{5}+5 n^{4}+n^{3}-3 n^{2}-2 n-2\right)}{4(n+1)^{3}(n+2)(2 n+3)} z_{c}^{2 n+5} \frac{n\left(n^{2}-3\right)}{4(n+1)(n+3)} z_{c}^{2 n+6}\right], \\
& W_{5}=h\left[\frac{(n+6)}{12(n+3)(n+4)} z_{c}^{2}-\frac{n(n+5)}{12(n+1)(n+3)} z_{c}^{3}+\frac{(n-1)(n+4)}{24(n+1)} z_{c}^{4}\right. \\
& -\frac{(n+3)}{12(n+1)} z_{c}^{6}-\frac{(n+2)}{24} z_{c}^{7}-\frac{n\left(n^{2}-3\right)}{12(n+1)} z_{c}^{n+4} \\
& -\frac{\left(n^{4}+2 n^{3}-5 n^{2}-6 n+4\right)}{8(n+1)(n+3)} z_{c}^{n+5}-\frac{n\left(n^{2}-3\right)}{4(n+1)} z_{c}^{n+7} \\
& \left.+\frac{n(n+3)\left(n^{4}+8 n^{3}+13 n^{2}-12 n-6\right)}{16(n+1)(n+4)(n+5)} z_{c}^{n+7}\right]
\end{aligned}
$$




$$
\begin{aligned}
W_{6}=h[- & \frac{n(2 n+5)}{4(n+2)(n+3)(2 n+3)} z_{c}^{2}+\frac{n^{2}}{4(n+1)^{2}} z_{c}^{3} \\
& -\frac{n(n-1)(2 n+3)}{8(n+1)(2 n+1)} z_{c}^{4}+\frac{n\left(n^{4}+3 n^{3}-3 n^{2}-7 n+2\right)}{8(n+1)^{2}(n+2)} z_{c}^{n+4} \\
& -\frac{\left(n^{3}+n^{2}-4 n+4\right)}{8(n+3)} z_{c}^{n+5}+\frac{n}{8} z_{c}^{n+6} \\
& \left.\quad-\frac{(n+2)\left(2 n^{2}+n-2\right)}{8(n+1)^{2}(2 n+1)(2 n+3)} z_{c}^{2 n+5}+\frac{n^{2}\left(n^{2}-3\right)}{8(n+1)(n+2)} z_{c}^{2 n+6}\right] \\
W_{7}=h[ & \frac{n(n+6)\left(n^{2}-3\right)}{12(n+1)(n+2)(n+3)(n+4)} z_{c}^{n+2}+\frac{n^{2}(n+5)\left(n^{2}-3\right)}{12(n+1)^{2}(n+2)(n+3)} z_{c}^{n+3} \\
+ & \frac{n(n-1)(n+4)\left(n^{2}-3\right)}{24(n+1)^{2}(n+2)} z_{c}^{n+4}-\frac{n\left(n^{2}-3\right)}{12(n+1)^{2}(n+2)} z_{c}^{n+5}+\frac{n\left(n^{2}-3\right)}{12(n+1)^{2}} z_{c}^{n+6} \\
& -\frac{n\left(n^{2}-3\right)}{24(n+1)} z_{c}^{n+7}-\frac{n^{2}\left(n^{2}-3\right)}{12(n+1)^{2}(n+2)} z_{c}^{2 n+4} \\
& +\frac{n\left(n^{2}-3\right)\left(n^{4}+2 n^{3}-5 n^{2}-6 n+4\right)}{8(n+1)^{2}(n+2)(n+3)} z_{c}^{2 n+5}+\frac{n\left(n^{2}-3\right)\left(n^{4}+6 n^{3}+7 n^{2}-6 n-4\right)}{8(n+1)^{2}(n+2)(n+3)(n+4)} z_{c}^{2 n+6} \\
& \left.-\frac{n^{2}\left(n^{2}-3\right)^{2}}{24(n+1)^{2}(n+2)} z_{c}^{2 n+7}\right] .
\end{aligned}
$$

\section{Acknowledgment}

The present work is supported by the research university grant of Universiti Sains Malaysia, Malaysia (Grant no.: 1001/PMATHS/811177).

\section{References}

[1] S. Agarwal and G. Jayaraman, "Numerical simulation of dispersion in the flow of power law fluids in curved tubes," Applied Mathematical Modelling, vol. 18, no. 9, pp. 504-512, 1994.

[2] V. Balasubramanian, G. Jayaraman, and S. R. K. Iyengar, "Effect of secondary flows on contaminant dispersion with weak boundary absorption," Applied Mathematical Modelling, vol. 21, no. 5, pp. 275285, 1997.

[3] G. N. Mercer and A. J. Roberts, "A centre manifold description of contaminant dispersion in channels with varying flow properties," SIAM Journal on Applied Mathematics, vol. 50, no. 6, pp. 1547-1565, 1990.

[4] G. I. Taylor, "Dispersion of soluble matter in solvent flowing slowly through a tube," Proceedings of the Royal Society A, vol. 219, pp. 186-203, 1953.

[5] E. J. Watson, "Diffusion in oscillatory pipe flow," Journal of Fluid Mechanics, vol. 133, pp. 233-244, 1983.

[6] R. Sankarasubramanian and W. N. Gill, "Unsteady convective diffusion with interphase mass transfer," Proceedings of the Royal Society A, vol. 333, pp. 115-132, 1973. 
[7] E. M. Lungu and H. K. Moffatt, "The effect of wall conductance on heat diffusion in duct flow," Journal of Engineering Mathematics, vol. 16, no. 2, pp. 121-136, 1982.

[8] S. Tsangaris and N. Athanassiadis, "Diffusion in oscillatory flow in a pipe," Zeitschrift für Angewandte Mathematik und Mechanik, vol. 65, p. 252, 1985.

[9] M. K. Sharp, R. D. Kamm, A. H. Shapiro, E. Kimmel, and G. E. Karniadakis, “Dispersion in a curved tube during oscillatory flow," Journal of Fluid Mechanics, vol. 223, pp. 537-563, 1991.

[10] A. Ramachandra Rao and K. S. Deshikachar, "An exact analysis of unsteady convective diffusion in an annulus pipe," Zeitschrift für angewandte Mathematik und Mechanik, vol. 67, no. 3, pp. 189-195, 1987.

[11] B. S. Mazumder and S. K. Das, "Effect of boundary reaction on solute dispersion in pulsatile flow through a tube," Journal of Fluid Mechanics, vol. 239, pp. 523-549, 1992.

[12] M. K. Sharp, "Shear-augmented dispersion in non-Newtonian fluids," Annals of Biomedical Engineering, vol. 21, no. 4, pp. 407-415, 1993.

[13] Y. Jiang and J. B. Grotberg, "Bolus contaminant dispersion in oscillatory tube flow with conductive walls," Journal of Biomechanical Engineering, vol. 115, no. 4, pp. 424-431, 1993.

[14] R. Smith and I. Walton, "A Burgers concentration dispersion equation," Journal of Fluid Mechanics, vol. 239, pp. 65-80, 1992.

[15] R. K. Dash, G. Jayaraman, and K. N. Mehta, "Shear augmented dispersion of a solute in a Casson fluid flowing in a Conduit," Annals of Biomedical Engineering, vol. 28, no. 4, pp. 373-385, 2000.

[16] W. N. Gill and R. Sankarasubramanian, "Exact analysis of unsteady convective diffusion," Proceedings of the Royal Society of London, vol. 316, no. 1526, pp. 341-350, 1970.

[17] D. S. Sankar and U. Lee, "Nonlinear mathematical analysis for blood flow in a constricted artery under periodic body acceleration," Communications in Nonlinear Science and Numerical Simulation, vol. 16, no. 11, pp. 4390-4402, 2011.

[18] S. U. Siddiqui, N. K. Verma, S. Mishra, and R. S. Gupta, "Mathematical modelling of pulsatile flow of Casson's fluid in arterial stenosis," Applied Mathematics and Computation, vol. 210, no. 1, pp. 1-10, 2009.

[19] C. Tu and M. Deville, "Pulsatile flow of Non-Newtonian fluids through arterial stenoses," Journal of Biomechanics, vol. 29, no. 7, pp. 899-908, 1996.

[20] D. S. Sankar, J. Goh, and A. I. M. Ismail, "FDM analysis for blood flow through stenosed tapered arteries," Boundary Value Problems, Article ID 917067, 16 pages, 2010.

[21] P. Chaturani and V. R. Ponnalagar Samy, "A study of non-Newtonian aspects of blood flow through stenosed arteries and its applications in arterial diseases," Biorheology, vol. 22, no. 6, pp. 521-531, 1985.

[22] D. S. Sankar and U. Lee, "Mathematical modeling of pulsatile flow of non-Newtonian fluid in stenosed arteries," Communications in Nonlinear Science and Numerical Simulation, vol. 14, no. 7, pp. 2971-2981, 2009.

[23] N. Iida, "Influence of plasma layer on steady blood flow in micro vessels," Japanese Journal of Applied Physics, vol. 17, pp. 203-214, 1978.

[24] Y. C. Fung, Biodynamics: Circulation, Springer, New York, NY, USA, 1984. 


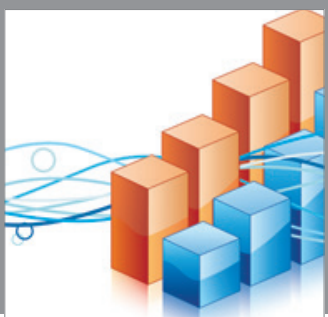

Advances in

Operations Research

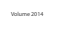

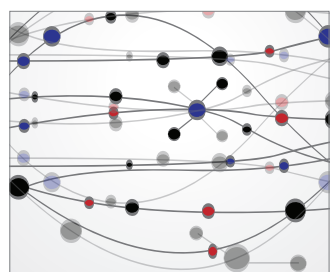

\section{The Scientific} World Journal
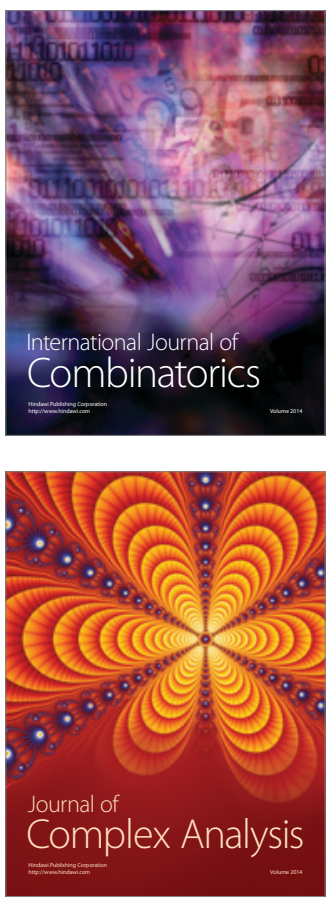

International Journal of

Mathematics and

Mathematical

Sciences
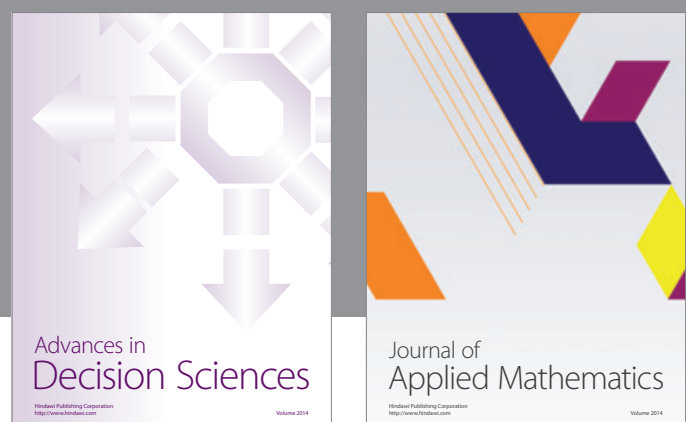

Journal of

Applied Mathematics
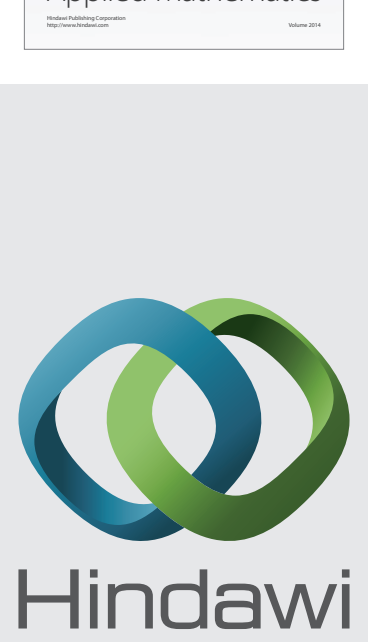

Submit your manuscripts at http://www.hindawi.com
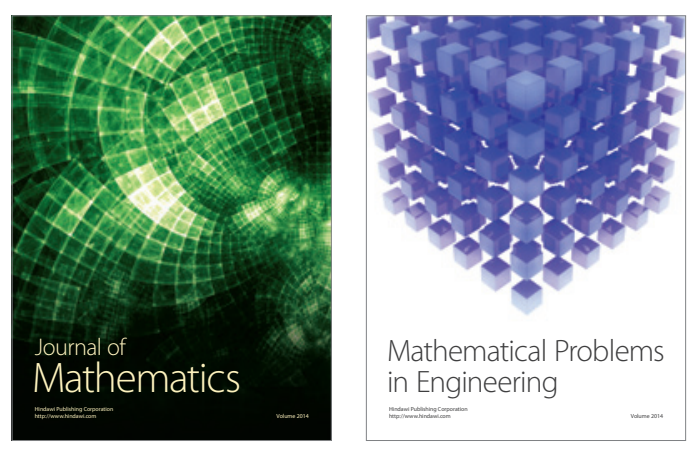

Mathematical Problems in Engineering
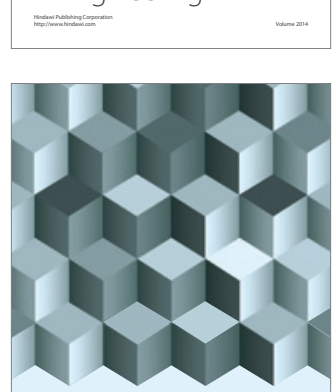

Journal of

Function Spaces
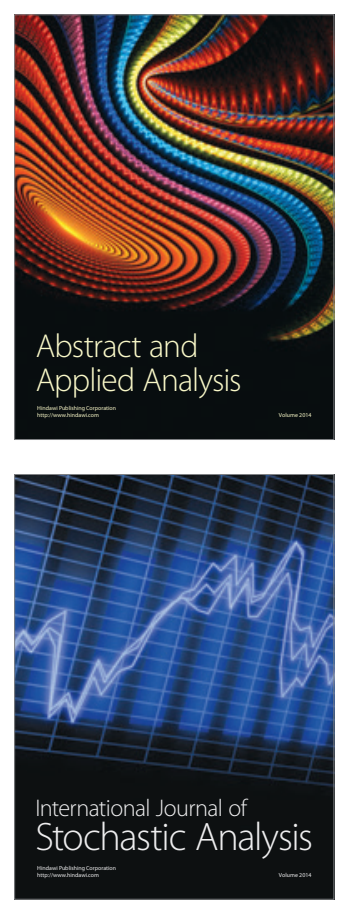

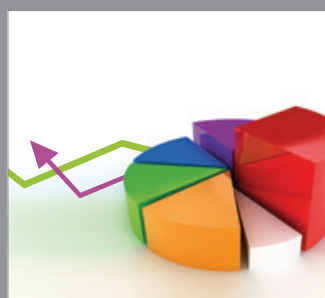

ournal of

Probability and Statistics

Promensencen
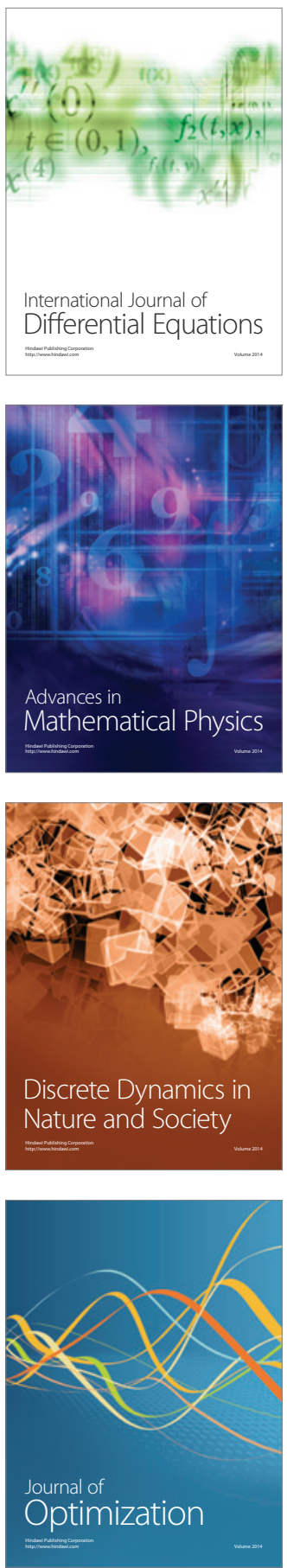